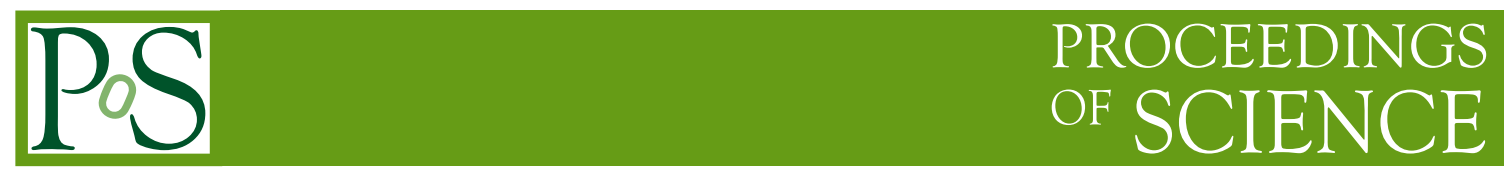

\title{
Northern sky Galactic Cosmic Ray anisotropy between 10-1000 TeV with the Tibet Air Shower Array
}

M. Amenomori ${ }^{1}$, X. J. Bi ${ }^{2}$, D. Chen ${ }^{3}$, T. L. Chen ${ }^{4}$, W. Y. Chen ${ }^{2}$, S. W. Cui ${ }^{5}$, Danzengluobu $^{4}$, L. K. Ding ${ }^{2}$, C. F. Feng ${ }^{6}$, Zhaoyang Feng ${ }^{* 2}$, Z. Y. Feng ${ }^{7}$, Q. B. Gou ${ }^{2}$, Y. Q. Guo ${ }^{2}$, H. H. He ${ }^{2}$, Z. T. He ${ }^{5}$, K. Hibino ${ }^{8}$, N. Hotta ${ }^{9}$, Haibing $\mathrm{Hu}^{4}$, H. B. $\mathrm{Hu}^{2}$, J. Huang ${ }^{2}$, H. Y. Jia ${ }^{7}$, L. Jiang ${ }^{2}$, F. Kajino ${ }^{10}$, K. Kasahara ${ }^{11}$, Y. Katayose ${ }^{12}$, C. Kato ${ }^{13}$, K. Kawata ${ }^{14}$, M. Kozai ${ }^{13}$, Labaciren ${ }^{4}$, G. M. Le ${ }^{15}$, A. F. Li ${ }^{16,6,2}$, H. J. Li ${ }^{4}$, W. J. Li ${ }^{2,7}$, C. Liu $^{2}$, J. S. Liu ${ }^{2}$, M. Y. Liu ${ }^{4}$, H. Lu ${ }^{2}$, X. R. Meng ${ }^{4}$, T. Miyazaki ${ }^{13}$, K. Mizutani ${ }^{11,17}$, K. Munakata ${ }^{13}$, T. Nakajima ${ }^{13}$, Y. Nakamura ${ }^{13}$, H. Nanjo ${ }^{1}$, M. Nishizawa ${ }^{18}$, T. Niwa ${ }^{13}$, M. Ohnishi ${ }^{14}$, I. Ohta ${ }^{19}$, S. Ozawa ${ }^{11}$, X. L. Qian ${ }^{6,2}$, X. B. Qu ${ }^{2}$, T. Saito ${ }^{20}$, T. Y. Saito ${ }^{21}$, M. Sakata ${ }^{10}$, T. K. Sako ${ }^{14}$, J. Shao ${ }^{2,6}$, M. Shibata $^{12}$, A. Shiomi ${ }^{22}$, T. Shirai ${ }^{8}$, H. Sugimoto ${ }^{23}$, M. Takita ${ }^{14}$, Y. H. Tan ${ }^{2}$, N. Tateyama ${ }^{8}$, S. Torii ${ }^{11}$, H. Tsuchiya ${ }^{24}$, S. Udo ${ }^{8}$, H. Wang ${ }^{2}$, H. R. $\mathbf{W u}^{2}$, L. Xue ${ }^{6}$, Y. Yamamoto ${ }^{10}$, K. Yamauchi ${ }^{12}$, Z. Yang ${ }^{2}$, S. Yasue ${ }^{25}$, A. F. Yuan ${ }^{4}$, T. Yuda ${ }^{14}$, L. M. Zhai ${ }^{2}$, H. M. Zhang ${ }^{2}$, J. L. Zhang ${ }^{2}$, X. Y. Zhang ${ }^{6}$, Y. Zhang ${ }^{2}$, Yi Zhang ${ }^{2}$, Ying Zhang ${ }^{2}$, Zhaxisangzhu ${ }^{4}$, X. X. Zhou ${ }^{7}$ 


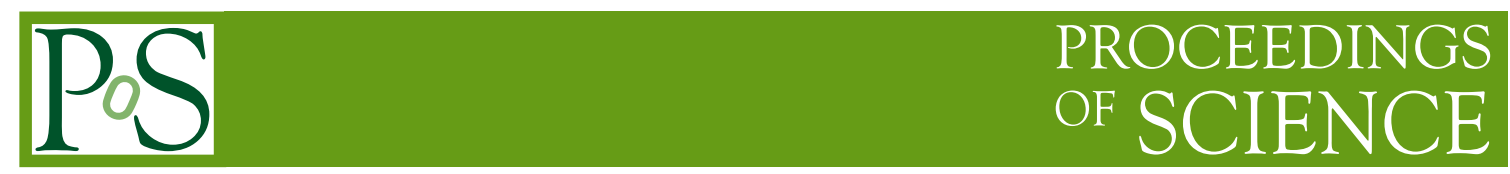

(The Tibet AS $\gamma$ Collaboration)

${ }^{1}$ Department of Physics, Hirosaki University, Hirosaki 036-8561, Japan

${ }^{2}$ Key Laboratory of Particle Astrophysics, Institute of High Energy Physics, Chinese Academy of Sciences, Beijing 100049, China

${ }^{3}$ National Astronomical Observatories, Chinese Academy of Sciences, Beijing 100012, China

${ }^{4}$ Department of Mathematics and Physics, Tibet University, Lhasa 850000, China

${ }^{5}$ Department of Physics, Hebei Normal University, Shijiazhuang 050016, China

${ }^{6}$ Department of Physics, Shandong University, Jinan 250100, China

${ }^{7}$ Institute of Modern Physics, SouthWest Jiaotong University, Chengdu 610031, China

${ }^{8}$ Faculty of Engineering, Kanagawa University, Yokohama 221-8686, Japan

${ }^{9}$ Faculty of Education, Utsunomiya University, Utsunomiya 321-8505, Japan

${ }^{10}$ Department of Physics, Konan University, Kobe 658-8501, Japan

${ }^{11}$ Research Institute for Science and Engineering, Waseda University, Tokyo 169-8555, Japan

${ }^{12}$ Faculty of Engineering, Yokohama National University, Yokohama 240-8501, Japan

${ }^{13}$ Department of Physics, Shinshu University, Matsumoto 390-8621, Japan

${ }^{14}$ Institute for Cosmic Ray Research, University of Tokyo, Kashiwa 277-8582, Japan

${ }^{15}$ National Center for Space Weather, China Meteorological Administration, Beijing 100081, China

${ }^{16}$ School of Information Science and Engineering, Shandong Agriculture University, Taian 271018, China

${ }^{17}$ Saitama University, Saitama 338-8570, Japan

${ }^{18}$ National Institute of Informatics, Tokyo 101-8430, Japan

${ }^{19}$ Sakushin Gakuin University, Utsunomiya 321-3295, Japan

${ }^{20}$ Tokyo Metropolitan College of Industrial Technology, Tokyo 116-8523, Japan

${ }^{21}$ Max-Planck-Institut für Physik, München D-80805, Deutschland

${ }^{22}$ College of Industrial Technology, Nihon University, Narashino 275-8576, Japan

${ }^{23}$ Shonan Institute of Technology, Fujisawa 251-8511, Japan

${ }^{24}$ Japan Atomic Energy Agency, Tokai-mura 319-1195, Japan

${ }^{25}$ School of General Education, Shinshu University, Matsumoto 390-8621, Japan $\dagger$

E-mail: fengzydihep.ac.cn

We report on the observation of the large-scale sidereal anisotropy of Galactic Cosmic Rays (GCRs) between $10 \mathrm{TeV}$ and $1 \mathrm{PeV}$, with the data collected by the Tibet Air Shower experiment between October 1995 and February 2010. The method to estimate the energy of GCRs energy is improved, so that the data with zenith angle up to 60 degrees can be used. The two-dimensional intensity map with declination between -30 degree and 90 degree at $300 \mathrm{TeV}$ is well connected with IceCube's observation at $400 \mathrm{TeV}$ in 2012, which gives a global picture about the anisotropy at this energy range. A new struction on the energy dependence of the first harmonic coefficients of the large scale anisotropy is revealed above $100 \mathrm{TeV}$.

The 34th International Cosmic Ray Conference,

30 July- 6 August, 2015

The Hague, The Netherlands

\footnotetext{
* Speaker.

${ }^{\dagger}$ The collaborative experiment of the Tibet Air Shower Arrays has been performed under the auspices of the Min-
} 


\section{Introduction}

The arrival direction of Galactic Cosmic Rays (GCRs) is nearly isotropic due to deflections in the Galactic Magnetic Field (GMF). However, observations of ground-based experiments in both the northern and the southern hemispheres show that there exists a slight anisotropy with a relative amplitude in the order of $10^{-4}$ to $10^{-3}$ on the overall isotropic background between $10 \mathrm{GeV}$ and several hundreds of $\mathrm{TeV}$ (see figure $\square$ and its references).

Due to the low flux of cosmic rays in the primary energy range of several hundreds $\mathrm{TeV}$ up to $10 \mathrm{PeV}$, only few statistically significant anisotropies were reported from ground-based airshower experiments. EAS-TOP experiment published an anisotropy observation at $E_{0} \sim 200 \mathrm{TeV}$ [四], and later, with more data, reported a sharp increase in the anisotropy for primary energies of $\sim 370 \mathrm{TeV}[[]]$. At higher energy from 0.7 to $6 \mathrm{PeV}$, no hints for anisotropy in the right ascension distributions were found by the KASCADE experiment [3]. Recently, IceCube experiment reported an observation of an anisotropy at $400 \mathrm{TeV}$ [䧃] in the southern sky, which was confirmed by Ice-Top experiment [5] later. Furthermore, IceTop found the anisotropy persists to PeV energies [ [0].

In our previous work [鴫, the Tibet AS $\gamma$ collaboration reported the sidereal anisotropy at multi$\mathrm{TeV}$ with the data taken between February 1997 and October 2005. When we were trying to search for $100 \mathrm{TeV} \gamma$ rays emission [ $[$ ], we got some hints of $300 \mathrm{TeV}$ cosmic ray anisotropy with the data only collected by Tibet AS array between Oct. 2000 and Dec.2008 .With data collected between Oct. 1995 and Feb. 2010, we report on the observation of the large-scale sidereal anisotropy of Galactic Cosmic Rays (GCRs) at median energy of $\sim 300 \mathrm{TeV}$ [ [ ] ] . A new anisotropy structure at $300 \mathrm{TeV}$ is revealed, which significantly deviates from the picture of cosmic ray isotropic intensity and is different with previous anisotropy observation at Multi-TeV energy and is consist with the observation of IceCube at $400 \mathrm{TeV}$ [䧃].

In this work, the method to estimate the energy of GCRs energy is improved, and the data with zenith angle up to 60 degrees can be used, which means that the anisotropy with declination from -30 degree to 90 degree can be measured. We report on the observation of the large-scale sidereal anisotropy of Galactic Cosmic Rays (GCRs) between $10 \mathrm{TeV}$ and $1 \mathrm{PeV}$, with the data collected by the Tibet Air Shower experiment between October 1995 and February 2010.

\section{Analysis}

\subsection{Experiment and Data reconstruction}

Tibet AS $\gamma$ experiment is located at Yangbajing in Tibet, China $\left(90.522^{\circ} \mathrm{E}, 30.102^{\circ} \mathrm{N}, 4300 \mathrm{~m}\right.$ a.s.1., $606 \mathrm{~g} / \mathrm{cm}^{2}$ ). The effective area of the Tibet AS array has been gradually enlarged in several steps, by adding the same-type plastic scintillation detectors with an area of $0.5 \mathrm{~m}^{2}$ to the preceding Tibet-I, II and III arrays. In addition, the performance was improved by adding detectors for a more compact array. The Tibet-II array consists of 221 detectors with a 15-m grid covering in total 36900 $m^{2}$. It started operation in October 1995. The current Tibet-III was upgraded to a dense array with

istry of Science and Technology of China and the Ministry of Foreign Affairs of Japan. The speaker Zhaoyang Feng is supported by the Natural Sciences Foundation of China (No.11135010, No. 11405182), and by the Chinese Academy of Sciences (524014IHEPZZBS301). 
7.5-m grid in 1999 and 2003 []. The trigger rates are $\sim 230 \mathrm{~Hz}$ and $\sim 1700 \mathrm{~Hz}$ for the Tibet-II and III arrays, respectively.

In order to keep the data performance stable, we keep the same form of the data throughout the observation period from October 1995 to February 2010 by reconstructing air showers obtained from the detector configuration of the Tibet-II array completed in 1995 unchanged for the Tibet-III array. So that the full data sample taken by Tibet II and Tibet III array can be used in the present analysis.

Then the traditional shower reconstruction procedure is applied to get all the parameters of one shower, such as core position, direction $(\theta, \phi)$ and shower size $\sum \rho_{F T}$, which is the sum of the number of particles per $\mathrm{m}^{2}$ counted by all the fast-timing (FT) detector. The following criteria is applied to select well reconstructed events: (1) each AS event should fire four or more detectors recording 1.25 or more particles, (2) the AS core position should be located inside the array,

\subsection{Estimation of Cosmic-ray Energy}

The air showers reaching Tibet AS array with a large zenith angle $\theta$ have to cross a larger slant depth than vertically propagating showers. This introduces a zenith angle dependence of the relation between $\sum \rho_{F T}$ and the primary particle energy. In most of the previous works of

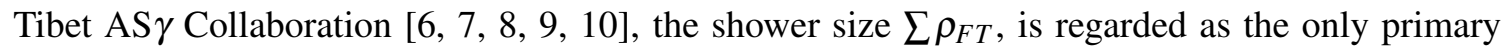
energy reference. The zenith angle dependence of the relation between $\sum \rho_{F T}$ and the primary particle energy is not considered. This approximation works well in small zenith angle $\left(\theta<\sim 40^{\circ}\right)$, considering the natural fluctuations that arise in the development of the extensive air showers and limited resolution of the primary energy.

In this work, we try to explore the anisotropy with declination down to -30 degree. Then the shower with zenith angle up to 60 degree would be used. The zenith angle dependence of the relation between $\sum \rho_{F T}$ and the primary particle energy would play an important role in this analysis. Therefore, a two-dimensional cut in $\sum \rho_{F T}$ and $\theta$ is used.

The uncertainty in the cosmic-ray energy estimation has been modeled with a full simulation of cosmic-ray interactions in the atmosphere using a full Monte Carlo (MC) simulation. It is performed for the air shower development in the atmosphere by CORSIKA (version 6.204) [W]] with QGSJET01c being chosen as the hadronic interaction model and for the detector response by Epics (version 8.65) [ए2]. together with the composition and the spectrum of primary cosmic rays as described in [[13]].

Figure $\mathbb{0}$ shows the distribution from simulation of the cosmic-ray primary particle energy with respect to $\sum \rho_{F T}$ as a function of $\sec \theta$. The figure shows that for a given range of $\sum \rho_{F T}$, small zenith events (i.e., $\sec \theta \sim 1$ ) are dominated by $\operatorname{cosmic}$ rays with lower average energy than large zenith angle events (i.e., $\sec \theta \sim 2$ ). We identified regions of constant primary energy in $\left(\sum \rho_{F T}, \sec \theta\right)$, delimited with the dashed lines in Figure W, in order to select five event samples (15 $\mathrm{TeV}, 50 \mathrm{TeV}, 100 \mathrm{TeV} 300 \mathrm{TeV}$ and $1 \mathrm{PeV}$ ) at energies with minimal overlap.

Figure $\square$ shows the simulated primary energy distributions for the five event samples. The uncertainty of the primary energy estimate is dominated by the fluctuations in the air showers. The number of events in different energy bands are $2.33 \times 10^{10}(15 \mathrm{TeV}), 3.97 \times 10^{9}(50 \mathrm{TeV})$, $1.96 \times 10^{9}(100 \mathrm{TeV}), 2.71 \times 10^{8}(300 \mathrm{TeV}), 5.72 \times 10^{7}(1 \mathrm{PeV})$. 


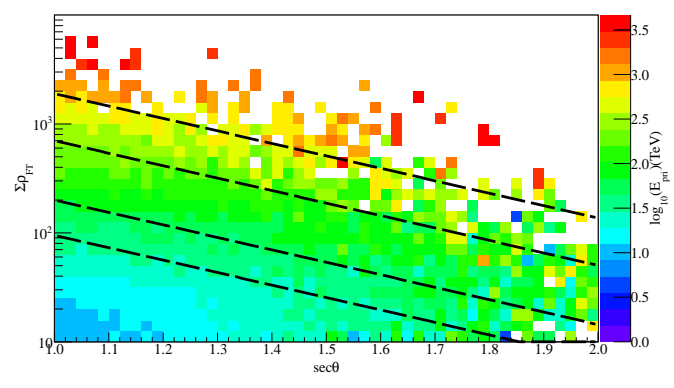

Figure 1: Average logarithm of the cosmic-ray primary energy as a function of $\sum \rho_{F T}$ and zenith angle, as obtained from simulation. The Y-axis is the $\sum \rho_{F T}$, the $\mathrm{X}$-axis is the secant of the reconstructed zenith angle of the event while the color scale is the mean of the logarithm of the cosmic-ray primary energy for each bin obtained from simulation in $\mathrm{TeV}$. The dashed lines are the borders of different energy bands (15 TeV, 50 $\mathrm{TeV}, 100 \mathrm{TeV}, 300 \mathrm{TeV}$ and $1 \mathrm{PeV})$.

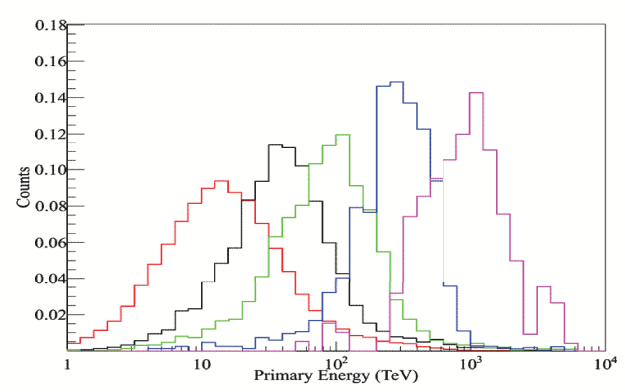

Figure 2: Normalized counts of events vs. the primary energy (in TeV) plotted for the five selected energy samples. The five energy bands are: $15 \mathrm{TeV}$ ( red line), $50 \mathrm{TeV}$ (black line), $100 \mathrm{TeV}$ (green line), $300 \mathrm{TeV}$ (blue line) and $1 \mathrm{PeV}$ (pink line). The energy distributions were determined using a full simulation.

\subsection{Analysis method and the first harmonic function fitting}

The All-Distance Equi-Zenith Angle Method [ए]], which was also used in our previous work [目], is applied to the data set, in order to reconstruct a possible large-scale anisotropy. Details about the analysis method are in [ए]]. In this work, the sky is divided into cells with bin size of $1^{\circ}$ in both zenith direction between $0^{\circ}$ and $60^{\circ}$ and azimuth direction between $0^{\circ}$ and $360^{\circ}$, and the sky in equatorial coordinates is divided into cells of $2^{\circ} \times 2^{\circ}$ between $0^{\circ}$ and $360^{\circ}$ in the right ascension $(\alpha)$ and between $-30^{\circ}$ and $90^{\circ}$ in the declination $(\delta)$. The smoothing search applied is from 5 to 30 degree.

To quantify the scale of the anisotropy, the one-dimensional (1D) profile of the sidereal anisotropy is created by projecting all the relative intensities in the declination range from $-30^{\circ}$ to $90^{\circ}$ onto the right ascension coordinate. We divide the right ascension into 18 bins. And then the $1 \mathrm{D}$ profile of the anisotropy is fitted by a harmonic function in the form of

$$
R(\alpha)=1+A_{1} \cos \left(\alpha-\phi_{1}\right),
$$

where $R(\alpha)$ denotes the relative intensity of cosmic ray at right ascension $\alpha, A_{1}$ is the amplitude of the 1 st harmonic term, $\phi_{1}$ is the phase of the 1 st harmonic term, at which the 1 st harmonic function reaches its maximum. 


\section{Results}

\subsection{Sidereal Anisotropy map at $300 \mathrm{TeV}$}

In order to enlarge the data statistic of $300 \mathrm{TeV}$ energy band, we merge the $300 \mathrm{TeV}$ energy band and $1 \mathrm{PeV}$ energy band in figure $\mathrm{W}$ as the new $300 \mathrm{TeV}$ energy band. The log-median energy of sample is still $\sim 300 \mathrm{TeV}$ and event number is $3.28 \times 10^{8}$.

Figure [ 3 shows the significance map and the relative intensity maps with an optimized smoothing of $30^{\circ}$. The anisotropy structure at $300 \mathrm{TeV}$ is revealed, which is similar to the result in [8].

For the significance map, two regions were identified to be significant. The first region is an excess at $\left(\alpha=263^{\circ}, \delta=11^{\circ}\right)$ with a significance of $7.2 \sigma$ and an optimized smoothing of $30 \mathrm{deg}$, and the second region is a deficit at $\left(\alpha=93^{\circ}, \delta=-25^{\circ}\right)$ with a significance of $-5.8 \sigma$. Note that these are the pre-trial significance values. We conservatively estimate a trial factor by assuming that all scans give statistically independent results. Since the search for this excess is performed over about $60 \times 180$ cells, and across 24 different smoothing radii in total, there is a trials factor of at most $2.59 \times 10^{5}$. After correcting for the trials, only the excess remained significant beyond the $5 \sigma$ level, with a post-trial significance value of $\sim 5.3 \sigma$.

Duo to the fact that the detector acceptance decreases with larger zenith angle., the maximum relative intensity region is not located at the most significance excess region. The character of the relative intensity map is similar to the significant map, an excess region $\left(\alpha=269^{\circ}, \delta=-13^{\circ}\right)$ at with an maximum relative intensity of $1+1.38 \times 10^{-3}$ and a deficit region at $\left(\alpha=87^{\circ}, \delta=-29^{\circ}\right)$ with an minmum relative intensity of $1-1.80 \times 10^{-3}$. Both the excess region and the deficit region the quite consistent with the observation by the IceCube experiment at $400 \mathrm{TeV}$ [团] in space. This result gives a global picture about the GCRs sidereal anisotropy at several hundreds of TeV.

Figure $B$ also shows the projection in right ascension of the cosmic ray relative intensity with statistical uncertainties. In this paper, the correlation among the bins is carefully considered when calculating the statistical errors in each bin and fitting the data by a harmonic function. We want to point out that if the correlation among the bins is not considered, it would results in a smaller statistical error of the fitting parameters. The blue line indicates the fit of eq. fitting parameters are shown in the figure. The significance of the amplitude measure is $5.6 \sigma$, which prove that this measurement is quite significant. The reduced $\chi^{2}$ values is $26.7 / 16$, which means that the first harmonic function can describe the 1D projection well.

\subsection{Transition of CR sidereal anisotropy between 10-1000 TeV}

Figure 9 shows the sidereal anisotropy transition between $10 \mathrm{TeV}$ and $1 \mathrm{PeV}$. At $15 \mathrm{TeV}$ and $50 \mathrm{TeV}$, the tail-in and loss-cone features[圂] are observed with very high significance, and the anisotropy component around the Cygnus region also can be seen. At $100 \mathrm{TeV}$, the excess and deficit region of the anisotropy map start to move, and the intensity change a little. At $300 \mathrm{TeV}$ and $1 \mathrm{PeV}$, the anisotropy maps are distinctly different with the maps at multi-TeVs. We can see the varied phases of the one dimensional projection in right ascension, as a function of the primary cosmic ray energies.

Figure 5 shows the amplitude and phase of the best-fit first harmonic function fit by the Tibet AS array, together with observations by other deep underground muon experiments and extensive 


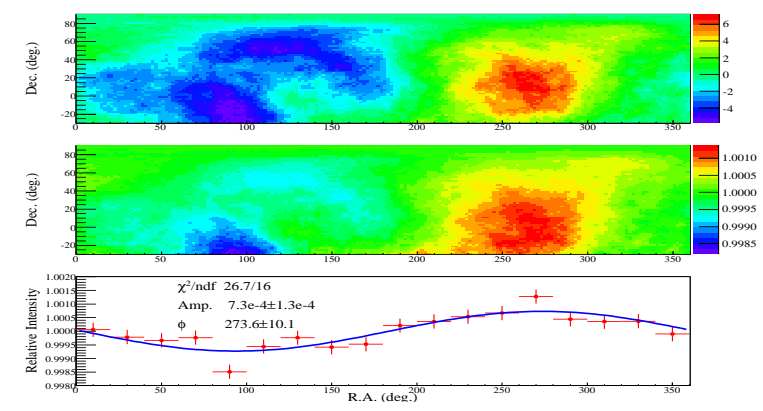

Figure 3: Large scale sidereal anisotropy measurement at $300 \mathrm{TeV}$ by the Tibet AS Array with an optimized smoothing of $30^{\circ}$. The top figure shows the significance map. The middle figure is the relative intensity map. The bottom figure shows the one dimensional projection in right ascension $\alpha$ of the two-dimensional cosmic ray map with declination between $-30^{\circ}$ and $90^{\circ}$. The blue line corresponds to the first harmonic fit to the data.

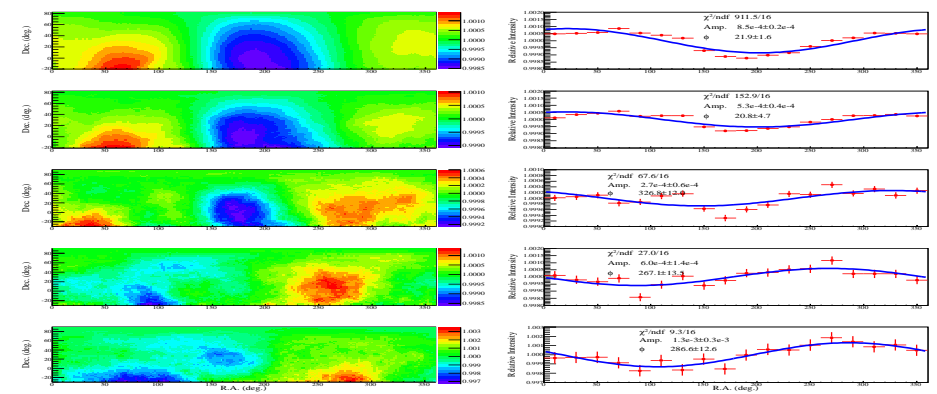

Figure 4: Anisotropy transition between $10 \mathrm{TeV}$ and $1 \mathrm{PeV}$. Left panel shows the celestial CR intensity map for different representative CR energies, $15 \mathrm{TeV}, 50 \mathrm{TeV}, 100 \mathrm{TeV}, 300 \mathrm{TeV}$ and $1 \mathrm{PeV}$ from up to down, with an smoothing of $30^{\circ}$. The vertical color bin width is changed for different statistics, all for the relative $\mathrm{CR}$ intensity. The right panel shower the corresponding one dimensional projection in right ascension $\alpha$ of the two-dimensional cosmic ray map with declination between $-30^{\circ}$ and $90^{\circ}$. The blue line corresponds to the first harmonic fit to the data.

air shower array experiments. The results in this work agree with other experiments in both amplitude and phase. From Figure [, we can see that a new struction on the energy dependence of the first harmonic coefficients of the large scale anisotropy is revealed above $100 \mathrm{TeV}$, which gives a new hints about the origin and propagation of GCRs. The interpretation of the structure is under studied.

Finally, as a check of the analysis method and the data sample, the same analysis were performed using the solar time frame, the anti-sidereal time frame and the ext-sidereal time frame. The observed solar time anisotropy agrees with the expected Compton-Getting (CG) effect[[प]] well within statistical error due to the terrestrial orbital motion around the Sun. The observed antisidereal time anisotropy show no significant observed amplitude in the anti-sidereal time, which insures the reliability of the anisotropy observed in sidereal time. Because of the limited pages, all these results are not showed in this paper. 

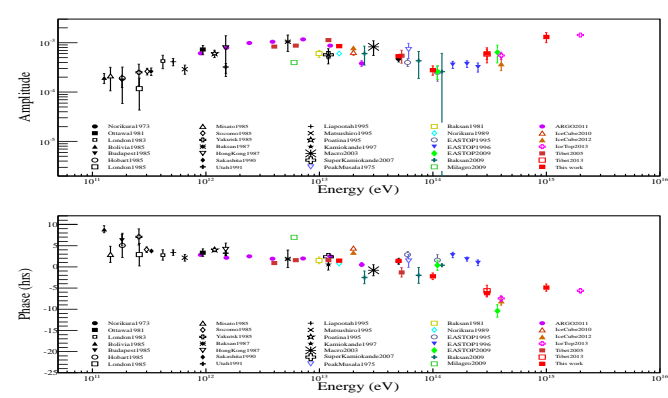

Figure 5: Anisotropy of the cosmic-ray primaries obtained by underground muon observations and extensive air shower array experiments. The amplitude (top) and phase (bottom) of the first Fourier harmonics in the sidereal-time variation are plotted as a function of the primary cosmic-ray energies.

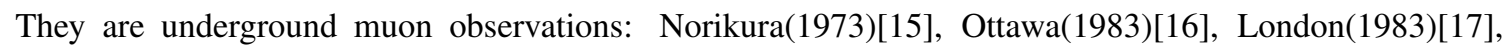

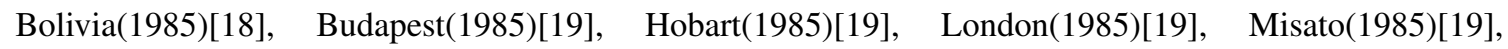

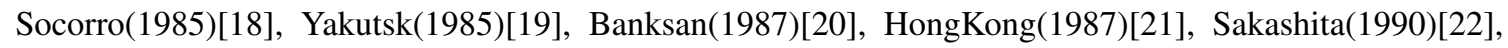
Utah(1991)[[23], Liapootah(1995)[24], Matsushiro(1995)[25], Poatina(1995)[26], Kamiokande(1997)[27], $\operatorname{Marco}(2003)$ [28], SuperKamiokande(2007)[ㅁ], and air shower array experiments: PeakMusala(1975)[BD], Baksan(1981)[B]], Norikura(1989)[B2], EASTOP(1995,1996,2009)[B3], 四, ㅁ], Baksan(2009)[B4],

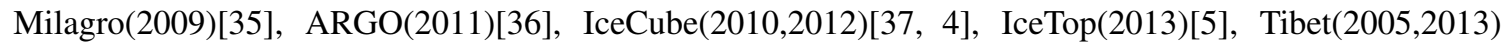
[B8, [1] and this work.

\section{Conclusion}

In this work, to explore the anisotropy with declination down to -30 degree, a two-dimensional cut in $\sum \rho_{F T}$ and $\theta$ is used to improve the primary energy estimation of cosmic rays. Using the data collected by the Tibet Air Shower experiment between October 1995 and February 2010, we report on the observation of the large-scale sidereal anisotropy of Galactic Cosmic Rays (GCRs) between $10 \mathrm{TeV}$ and $1 \mathrm{PeV}$ with declination from -30 degree to 90 degree. The two-dimensional intensity map with declination from -30 degree to 90 degree at $300 \mathrm{TeV}$ is well connected with IceCube's observation at $400 \mathrm{TeV}$ in 2012. A new struction on the energy dependence of the first harmonic coefficients of the large scale anisotropy is revealed above $100 \mathrm{TeV}$, which gives a new hints about the origin of GCRs, but The origin of this struction is unknown.

\section{References}

[1] Aglietta, M., et al. 1996, ApJ, 470, 501

[2] Aglietta, M., et al. 2009, ApJL, 692, L130

[3] Antoni, T., Apel, W. D., Badea, A. F., et al. 2004, ApJ, 604, 687

[4] Abbasi, R., Abdou, Y., Abu-Zayyad, T., et al. 2012, ApJL, 746, 33

[5] Aartsen, M. G., Abbasi, R., Abdou, Y., et al. 2013, ApJ, 765, 55

[6] Amenomori, M., et al. 2006, Science, 314, 439

[7] Zhaoyang Feng et al., Proceedings of the $31^{\text {st }}$ ICRC, Łódź 2009, ID0869

[8] M. Amenomori et al., Proceedings of the $33^{\text {rd }}$ ICRC, Rio de Janeiro 2013, ID0256 
[9] Amenomori, M. et al. 2003, ApJ, 598, 242

[10] Amenomori, M., et al. 2005, ApJ, 633, 1005

[11] Heck D, Knapp J, Capdevielle J N, Schatz G, Thouw T, 1998, Report FZKA 6019

[12] Kasahara K, http://cosmos.n.kanagawa-u.ac.jp/EPICSHome/index.html

[13] H órandel, J. R. 2003, Astropart. Phys., 19, 19

[14] Compton A. H., \& Getting, I. A. 1935, Physical Review, 47, 817

[15] S. Sakakibara et al., Proc. of the 13th International Cosmic Ray Conference, 2.1058 (1973)

[16] M. Bercovitch and S. P. Agrawal, Proc. of the 17th International Cosmic Ray Conference 10, 246 (1981).

[17] T. Thambyahpillai, Proc. of the 18th International Cosmic Ray Conference 3, 383 (1983).

[18] D. B. Swinson and K. Nagashima, Planet. Space Sci. 33, 1069 (1985).

[19] K. Nagashima et al., Planet. Space Sci. 33, 395 (1985).

[20] Y. M. Andreyev et al., Proc. of the 20th International Cosmic Ray Conference 2, 22 (1987).

[21] Y. W. Lee and L. K. Ng, Proc. of the 20th International Cosmic Ray Conference 2, 18 (1987).

[22] H. Ueno et al., Proc. of the 21st International Cosmic Ray Conference 6, 361 (1990).

[23] D. J. Cutler and D. E. Groom, Astrophys. J. 376, 322 (1991).

[24] K. Munakata et al., Proc. of the 24th International Cosmic Ray Conference 4, 639 (1995).

[25] S. Mori et al., Proc. of the 24th International Cosmic Ray Conference 4, 648 (1995).

[26] K. B. Fenton et al., Proc. of the 24th International Cosmic Ray Conference 4, 635 (1995).

[27] K. Munakata et al., Phys. Rev. D 56, 23 (1997).

[28] M. Ambrosio et al., Phys. Rev. D 67, 042002 (2003).

[29] Guillian, G., et al. 2007, PRD, 75, 062003

[30] T. Gombosi et al., Proc. of the 14th International Cosmic Ray Conference 2, 586 (1975).

[31] V. V. Alexeenko et al., Proc. of the 17th International Cosmic Ray Conference 2, 146 (1981).

[32] K. Nagashima et al., IL NUOVO CIMENTO VOL. 12 C, N. 6 Novembre-Dicembre 1989

[33] Aglietta, M., et al. Proc. of the 33th International Cosmic Ray Conference,2..800 (1995)

[34] V.V. Alekseenko, et al., Nuclear Physics B (Proc. Suppl.) 196 (2009) $1791 ́$ 182

[35] Abdo, A. A., et al. 2009, ApJ, 698, 2121

[36] R. Iuppa, arXiv:1112.2375,2011 Fermi Symposium, Roma., May. 9-12

[37] Abbasi, R. U., et al. 2010, ApJL, 718, L194

[38] M. Amenomori et al., ApJ, 626, L29-L32 (2005) 